\title{
Magnetic field wave equations for TM-modes in nonlinear optical waveguides
}

\author{
C.A.Stuart \\ Département de Mathématiques, EPFL, CH 1015 Lausanne
}




\section{Contents}

1 Introduction 1

2 Constitutive assumptions $\mathbf{5}$

2.1 Uni-axial medium . . . . . . . . . . . . . 5 5

2.2 Isotropic medium . . . . . . . . . . . . . . . . . . 9

2.3 Examples ...................... 11

3 Magnetic field wave equations 16

3.1 General TM-modes . . . . . . . . . . . . . . . . . 18

3.2 Planar TM-modes . . . . . . . . . . . . . . . . . . . . 19

3.3 Cylindrical TM-modes . . . . . . . . . . . . . . . 19

4 Existence of guided TM-modes 23

4.1 Guided planar TM-modes . . . . . . . . . . . . . . 23

4.2 Guided cylindrical TM-modes . . . . . . . . . . . 25

5 References $\quad 29$

\section{Introduction}

In CGS units, where $c$ is the s Maxwell's equations for a dielectric medium are [1],

$$
\begin{array}{cl}
\nabla \wedge E=-\frac{1}{c} \partial_{t} B \quad(1) & \nabla \wedge H=\frac{1}{c} \partial_{t} D \\
\nabla \cdot D=0 \quad(3) & \nabla \cdot B=0
\end{array}
$$

peed of light in a vacuum and the medium is charge-free. The fields $E, D, H$ and $B$ are treated as functions of Cartesian coordinates $(x, y, z, t) \in \Re^{4}$ and $e_{i}$ denotes the usual basis vector in $\Re^{3}$.

Assuming that the medium is non-magnetic we set $H \equiv B$. Then the remaining constitutive assumption about the medium should determine the electric displacement field, $D$, as a function of the electric field, $E$. In general, this is a complicated nonlinear relationship which for the moment we indicate roughly as $D=F(E)$.

The search for solutions of Maxwell's equations is usually based on the following electric field wave equation, 


$$
\nabla \wedge \nabla \wedge E=-\frac{1}{c^{2}} \partial_{t}^{2} F(E)
$$

which is obtained from (ME 1,2) and the constitutive relations $H=B$ and $D=F(E)$. Given a solution of this equation, the other fields can be defined so as to furnish a complete solution of Maxwell's equations. In cases where $E$ has a simple form (some TE-modes, for example) this is the most natural and straight forward way to proceed. However in other cases (some TMmodes, for example), the magnetic fields have a simpler form than the electric fields and it would seem advantageous to use the magnetic field as the basic unknown. In the case of linear isotropic materials the constitutive relations are $H=B$ and $D=\varepsilon(x, y, z) E$ where $\varepsilon$ is the dielectric constant. Thus $E=\frac{1}{\varepsilon} D$ and Maxwell's equations can be reduced to the study of the following magnetic field wave equation,

$$
\nabla \wedge \frac{1}{\varepsilon} \nabla \wedge H=-\frac{1}{c^{2}} \partial_{t}^{2} H
$$

Clearly anisotropic linear materials can be treated similarly using the dielectric tensor. For nonlinear materials (even isotropic ones), expressing $E$ as a function of $D$ is not such a simple matter since the constitutive relation expresses $\varepsilon$ as a function of $E$ and, perhaps for this reason, a purely magnetic field formulation of Maxwell's equations does not seem to have been used. Indeed, when comparing the study of TE-modes and TM-modes in planar optical waveguides, Stegeman describes the situation in the following way [[15], page 32]. "Extension of the analysis to TM nonlinear waves is complicated by the inherent structure of the fields; that is, they contain two electric field components $E_{x}(x)$ and $E_{z}(x)$ that are $\pi / 2$ out of phase with one another. Although much of the early work involved various approximations, this case has recently been solved exactly. In fact, it is necessary to start with Maxwell's equations and not a wave equation. (Rigorously, no simple nonlinear wave equations exists for this case.)" The first aim of this paper is to show how such a wave equation can be derived in quite a general context which is appropriate for many problems in nonlinear optics. The particular situation being referred to by Stegeman is treated in Section 4 where the nonlinear wave equation is a single second order ordinary differential equation.

In nonlinear optics the discussion is often restricted to monochromatic fields and the constitutive relation $D=F(E)$ is taken in a special, but still nonlinear, form. (See the beginning of Section 2.) In this paper we only 
deal with this situation in the case of a homogeneous uni-axial medium, but the approach could be extended to cover heterogeneous, fully anisotropic materials and to allow for absorption. The treatment of non-monochromatic fields would seem to be much more difficult.

In Section 2 we present a general form, (CR), of the constitutive relation that is widely used in the analysis of nonlinear optical waveguides, [31] and [16]-[18]. The main subject of that section concerns the inversion of this relationship so as to express $E$ as a function of $D$. Then in Section 3, we derive the nonlinear magnetic field wave equation that generalizes the linear one noted above. In particular, the special forms which are appropriate for the discussion of TM-modes propagating in planar and cylindrical wave-guides are presented. These configurations are of particular interest in nonlinear optics and previous work on them has been based on the system of two equations that arises in the electric field formulation [5]-[10] and [26][28], of the equations for TM-modes. An advantage of the present magnetic field formulation is that they are described by a single scalar second order (nonlinear) differential equation. For example, the equation (25) which we derive for cylindrical TM-modes has a relatively simple form compared to the equations $(10 \mathrm{a}, \mathrm{b})$ in [9] which arise in the EFWE formulation. A rigorous treatment of guidance for nonlinear TM-modes can be based on the equations derived in Section 3 and, in Section 4, we review what has been done so far.

Having given a summary of the contents of this paper, it is now appropriate to expand the earlier remarks about the nonlinear MFWE and about the study of TM-modes in nonlinear optics. The numerical approximation of a nonlinear MFWE has been undertaken recently; see for example [11] and [12]. However in that work the exact equation is never stated and the problem of expressing $\frac{1}{\varepsilon}$ as an explicit function of $H$ is not addressed. Instead, a two-step iterative procedure is used so as to avoid inverting the constitutive relation. Since our aim is to obtain qualitative, as well as quantitative, information about true solutions we need the explicit, exact form of the nonlinear MFWE. The only case that we know of where this has been attempted previously is in the work of Leung [13] in the special case of a planar waveguide. He does obtain an exact (integrable) equation for TM-modes using a magnetic field formulation by inverting the function $\varepsilon$ that appears in the isotropic version of the constitutive relation (CR). This does not lead to an inversion of the constitutive relation and his approach cannot be extended to more general situations. Even for planar waveguides, the equation which we formulate in Section 3 is simpler and more tractable. Apart from the papers that we have just mentioned, most of the work on TM-modes in planar or cylindrical waveguides has been based on a electric field formulation. A variety of ap- 
proaches to the planar case have been proposed [5]-[10], [24], [26]-[28]. They are applicable to various levels of generality of the constitutive relation, but they rely on the hypothesis that the medium is composed of a small number of homogeneous layers.

Acknowledgement This work was partly supported by a grant from the Swiss Office Fédérale de l'Education et de la Science for the project No OFES 93.0190 Variational Methods in Nonlinear Analysis, in collaboration with the European Union programme Human Capital and Mobility. 


\section{Constitutive assumptions}

All the e-m fields $E, D, H$ and $B$ which we consider can be expressed in the form

$$
F(x, y, z, t)=F_{T}(x, y) \cos (k z-\omega t)+F_{z}(x, y) \sin (k z-\omega t)
$$

where $F_{T}, F_{z}: \Re^{2} \longrightarrow \Re^{3}$ are such that $F_{T} \cdot e_{3}=0$ and $F_{z} \cdot e_{i}=0$ for $i=1,2$.

Thus the fields constitute monochromatic waves propagating in the direction of $e_{3}$ with the transverse and axial components of the fields being out of phase by a quarter of a cycle.

For fields of this type the constitutive relation which we adopt will be discussed in two steps. First we deal with a medium having uni-axial anisotropy, [31], and then we show the simplifications which occur when the medium is actually isotropic. In both cases we start from the standard form of constitutive assumption ([4], [17], [32]) which is usually adopted in the literature concerning nonlinear optical wave-guides, but the main purpose here is to introduce an equivalent formulation which enables us to derive a magnetic field wave equation with the aim of simplifying the treatment of TM-modes.

\subsection{Uni-axial medium}

To introduce the constitutive assumption for a homogeneous uniaxial material, we choose the orthonormal basis $\left\{e_{i}: i=1,2,3\right\}$ with $e_{3}$ in the direction of the axis of the medium.

(CR) $B=H$ and there exist two continuous functions $\varepsilon_{i}:[0 . \infty)^{2} \longrightarrow$ $(0, \infty)$ such that

$$
\begin{aligned}
& D_{T}(x, y)=\varepsilon_{1}\left(\left|E_{T}(x, y)\right|^{2} / 2,\left|E_{z}(x, y)\right|^{2} / 2\right) E_{T}(x, y) \\
& D_{z}(x, y)=\varepsilon_{2}\left(\left|E_{T}(x, y)\right|^{2} / 2,\left|E_{z}(x, y)\right|^{2} / 2\right) E_{z}(x, y)
\end{aligned}
$$

where $0<A<\varepsilon_{i}\left(s_{1}, s_{2}\right)$ for $i=1,2$ and for $s_{1}, s_{2} \geq 0$.

This means that with respect to the basis $\left\{e_{i}: i=1,2,3\right\}$ the dielectric response tensor is represented by the diagonal matrix $\left(\begin{array}{ccc}\varepsilon_{1} & 0 & 0 \\ 0 & \varepsilon_{1} & 0 \\ 0 & 0 & \varepsilon_{2}\end{array}\right)$ 
where the elements $\varepsilon_{i}$ are functions of the time-averages, $\left|E_{T}(x, y)\right|^{2} / 2$ and $\left|E_{z}(x, y)\right|^{2} / 2$, of the intensities of the transverse and axial components of the electric field $E$. Let us remark that the functions $\varepsilon_{i}$ also depend upon the frequency $\omega$ of $E$, but since we shall always treat $\omega$ as a fixed parameter we do not exhibit this dependence explicitly. Furthermore in a heterogeneous medium the elements $\varepsilon_{i}$ will also depend explicitly on $x, y$ and $z$. Again we do not deal with this case but we emphasize that all of the subsequent development extends to cover heterogeneous materials with only notational changes and some obvious requirements about the smoothness of the dependance on $(x, y, z)$.

According to $(\mathrm{CR})$ the dielectric response of the medium is determined by a function $\varepsilon=\left(\varepsilon_{1}, \varepsilon_{2}\right):[0, \infty)^{2} \longrightarrow(0, \infty)^{2}$ about which we now make some further assumptions.

(V) There is a potential $\varphi \in C^{1}\left([0, \infty)^{2}\right)$ such that $\varepsilon=\nabla \varphi$.

This property of the dielectric response is used explicitly in [7] where it is assumed that $\partial_{2} \varepsilon_{1}=\partial_{1} \varepsilon_{2}$ for all $s_{1}, s_{2} \geq 0$. When $(\mathrm{V})$ holds the potential, normalized so that $\varphi(0)=0$, is given by

$$
\varphi\left(s_{1}, s_{2}\right)=\int_{0}^{1} \varepsilon_{1}\left(t s_{1}, t s_{2}\right) s_{1}+\varepsilon_{2}\left(t s_{1}, t s_{2}\right) s_{2} \quad d t
$$

and we define an auxiliary function $\Phi$ by

$$
\Phi\left(s_{1}, s_{2}\right)=\varphi\left(s_{1}^{2} / 2, s_{2}^{2} / 2\right) .
$$

(C) We suppose that $\Phi \in C^{2}\left(\Re^{2}\right)$ and that $D^{2} \Phi(s)$ is positive definite for all $s \in \Re^{2}$.

The positive definitiveness of $D^{2} \Phi(s)$ implies that $\partial_{i}\left[\varepsilon_{i}\left(s_{1}^{2} / 2, s_{2}^{2} / 2\right) s_{i}\right]>0$ for $s \in \Re^{2}$ and $i=1,2$ and that $\Phi$ is strictly convex. Since $\Phi(0)=0$ and $\nabla \Phi(0)=0$, it follows that $\Phi(s)>0$ for all $s \in \Re^{2} \backslash\{0\}$. By (CR), $\left\|\nabla \Phi\left(s_{1}, s_{2}\right)\right\| \geq A \sqrt{\left(s_{1}^{2}+s_{2}^{2}\right)}$ for all $\left(s_{1}, s_{2}\right) \in \Re^{2}$ and so from the conclusions of Theorem 26.4 to Lemma 26.7 of [30] we find that $\nabla \Phi: \Re^{2} \longrightarrow \Re^{2}$ is a diffeomorphism and $[\nabla \Phi]^{-1}=\nabla \Phi^{*}$ where $\Phi^{*}$ is the Legendre transform of $\Phi$ which can be defined by

$$
\Phi^{*}(\tau)=\sup \left\{s . \tau-\Phi(s): s \in \Re^{2}\right\} \text { for } \tau=\left(\tau_{1}, \tau_{2}\right) \in \Re^{2} .
$$

From this formula we deduce that $\Phi^{*}(0,0)=0$ and $\Phi^{*}\left(s_{1}, s_{2}\right)=\Phi^{*}\left(\left|s_{1}\right|\right.$ , $\left.\left|s_{2}\right|\right)$. Since $\varepsilon_{1}$ and $\varepsilon_{2}>0$ we also have that $\nabla \Phi$ maps the four quadrants 
and half-axes onto themselves. Of course $\nabla \Phi\left(s_{1}, s_{2}\right)=\left(\begin{array}{l}\varepsilon_{1}\left(s_{1}^{2} / 2, s_{2}^{2} / 2\right) s_{1} \\ \varepsilon_{2}\left(s_{1}^{2} / 2, s_{2}^{2} / 2\right) s_{2}\end{array}\right)$ and we now show that $\nabla \Phi^{*}$ has a similar structure. From (C) we deduce that $\Phi^{*} \in C^{2}\left(\Re^{2}\right)$ and that $D^{2} \Phi^{*}(t)$ is positive definite for all $t \in \Re^{2}$. See Corollary 4.2 .10 of [29].

Define $\psi:[0, \infty)^{2} \longrightarrow \Re$ by

$$
\psi\left(t_{1}, t_{2}\right)=\Phi^{*}\left(\sqrt{2 t_{1}}, \sqrt{2 t_{2}}\right)
$$

so that

$$
\Phi^{*}\left(t_{1}, t_{2}\right)=\psi\left(t_{1}^{2} / 2, t_{2}^{2} / 2\right) .
$$

Clearly, $\psi \in C^{2}\left((0, \infty)^{2}\right)$ with $\partial_{i} \Phi^{*}\left(t_{1}, t_{2}\right)=\partial_{i} \psi\left(t_{1}^{2} / 2, t_{2}^{2} / 2\right) t_{i}$ and we define $\left(\gamma_{1}, \gamma_{2}\right) \in C^{1}\left((0, \infty)^{2}, \Re^{2}\right)$ by $\gamma_{i}=\partial_{i} \psi$. Then

$$
\begin{aligned}
& \left(\begin{array}{c}
t_{1} \\
t_{2}
\end{array}\right)=\left(\begin{array}{c}
\varepsilon_{1}\left(s_{1}^{2} / 2, s_{2}^{2} / 2\right) s_{1} \\
\varepsilon_{2}\left(s_{1}^{2} / 2, s_{2}^{2} / 2\right) s_{2}
\end{array}\right) \\
& \Leftrightarrow t=\nabla \Phi(s) \Leftrightarrow \nabla \Phi^{*}(t)=s \Leftrightarrow \\
& \left(\begin{array}{c}
s_{1} \\
s_{2}
\end{array}\right)=\left(\begin{array}{l}
\gamma_{1}\left(t_{1}^{2} / 2, t_{2}^{2} / 2\right) t_{1} \\
\gamma_{2}\left(t_{1}^{2} / 2, t_{2}^{2} / 2\right) t_{2}
\end{array}\right) .
\end{aligned}
$$

It follows that for $i=1,2$ and $t_{1}, t_{2}>0, \gamma_{i}\left(t_{1}, t_{2}\right)=$

$$
\begin{gathered}
1 / \varepsilon_{i}\left(\gamma_{1}\left(t_{1}, t_{2}\right)^{2} t_{1}, \gamma_{2}\left(t_{1}, t_{2}\right)^{2} t_{2}\right)= \\
1 / \varepsilon_{i}\left(\left[\partial_{1} \Phi^{*}\left(\sqrt{2 t_{1}}, \sqrt{2 t_{2}}\right)\right]^{2} / 2,\left[\partial_{2} \Phi^{*}\left(\sqrt{2 t_{1}}, \sqrt{2 t_{2}}\right)\right]^{2} / 2\right) .
\end{gathered}
$$

Using this formula, and recalling that $\nabla \Phi^{*}$ is continuous on $\Re^{2}$, we see that $\gamma_{i}$ can be extended by continuity to $[0, \infty)^{2}$.

Let us note an additional property of $\gamma_{i}$. Since $D^{2} \Phi^{*}(t)$ is positive definite for all $t \in \Re^{2}, 0<\partial_{i}^{2} \Phi^{*}\left(t_{1}, t_{2}\right)=\partial_{i}\left\{\gamma_{i}\left(t_{1}^{2} / 2, t_{2}^{2} / 2\right) t_{i}\right\}$ for $i=1,2$ and all $\left(t_{1}, t_{2}\right) \in \Re^{2}$.

Summarizing these remarks we can formulate the following result.

Theorem 1 Suppose that the dielectric response in (CR) has the properties $(V)$ and $(C)$. Let $\Phi^{*}$ be defined by (4). Then $\Phi^{*} \in C^{2}\left(\Re^{2}\right)$ and $D^{2} \Phi^{*}(t)$ is positive definite for all $t \in \Re^{2}$. Furthermore $\nabla \Phi^{*}\left(t_{1}, t_{2}\right)=\left(\begin{array}{c}\gamma_{1}\left(t_{1}^{2} / 2, t_{2}^{2} / 2\right) t_{1} \\ \gamma_{2}\left(t_{1}^{2} / 2, t_{2}^{2} / 2\right) t_{2}\end{array}\right)$ where $\left(\gamma_{1}, \gamma_{2}\right)$ has the following properties 
(H)

$$
\begin{array}{r}
\left(\gamma_{1}, \gamma_{2}\right) \in C^{1}\left((0, \infty)^{2}, \Re^{2}\right) \cap C\left([0, \infty)^{2}, \Re^{2}\right) \text { and for } i=1,2, \\
0<\gamma_{i}\left(t_{1}, t_{2}\right)<1 / A \\
\gamma_{i}\left(t_{1}^{2} / 2, t_{2}^{2} / 2\right)=1 / \varepsilon_{i}\left(s_{1}^{2} / 2, s_{2}^{2} / 2\right) \text { where } t_{i}=\varepsilon_{i}\left(s_{1}^{2} / 2, s_{2}^{2} / 2\right) s_{i} \\
\text { and } \partial_{i}\left\{\gamma_{i}\left(t_{1}^{2} / 2, t_{2}^{2} / 2\right) t_{i}\right\}>0 \text { for all }\left(t_{1}, t_{2}\right) \in \Re^{2}
\end{array}
$$

If the function $\varepsilon$ in the constitutive relation (CR) has the properties (V) and $(\mathrm{C})$, then $(\mathrm{CR})$ can be expressed in the following way where the function $\gamma$ has the properties $(\mathrm{H})$.

$$
\begin{aligned}
& (\mathbf{C R})^{*} B=H \text { and } \\
& \begin{array}{l}
E_{T}(x, y)=\gamma_{1}\left(\left|D_{T}(x, y)\right|^{2} / 2,\left|D_{z}(x, y)\right|^{2} / 2\right) D_{T}(x, y) \\
E_{z}(x, y)=\gamma_{2}\left(\left|D_{T}(x, y)\right|^{2} / 2,\left|D_{z}(x, y)\right|^{2} / 2\right) D_{z}(x, y)
\end{array}
\end{aligned}
$$

where $\gamma$ is obtained from $\varepsilon$ by the above construction.

Additional properties of the functions $\varepsilon_{i}$ can easily be interpreted in terms of the functions $\gamma_{i}$.

1. Noting that $\gamma_{1}\left(t_{1}, 0\right)=1 / \varepsilon_{1}\left(\gamma_{1}\left(t_{1}, 0\right)^{2} t_{1}, 0\right)$ and that $\gamma_{1}\left(t_{1}, 0\right)^{2} t_{1}$ is strictly increasing, we see that $\gamma_{1}\left(t_{1}, 0\right)$ is a strictly decreasing function of $t_{1}$ when $\varepsilon\left(s_{1}, 0\right)$ is a strictly increasing function of $s_{1}$.

2. Suppose that there exist $\alpha \geq 0$ and $A>0$ such that

$$
\varepsilon_{2}\left(s_{1}, s_{2}\right) / s_{2}^{\alpha} \rightarrow A \text { as } s_{2} \rightarrow \infty,
$$

uniformly for $s_{1}$ in bounded subsets of $[0, \infty)$.

Then it follows that

$$
\gamma_{2}\left(t_{1}, t_{2}\right) t_{2}^{\beta} \rightarrow B \text { as } t_{2} \rightarrow \infty,
$$

uniformly for $t_{1}$ in bounded subsets of $[0, \infty)$

where $\beta=\alpha /(1+2 \alpha)$ and $B=\left(2^{\alpha} / A\right)^{1 /(1+2 \alpha)}$.

Finally we remark that in the case of a heterogeneous medium the fundamental relation between $\varepsilon_{i}$ and $\gamma_{i}$ becomes

$$
\begin{aligned}
\gamma_{i}\left(x, y, z, t_{1}^{2} / 2, t_{2}^{2} / 2\right) & =1 / \varepsilon_{i}\left(x, y, z, s_{1}^{2} / 2, s_{2}^{2} / 2\right) \\
\text { where } t_{i} & =\varepsilon_{i}\left(x, y, z, s_{1}^{2} / 2, s_{2}^{2} / 2\right) s_{i} .
\end{aligned}
$$




\subsection{Isotropic medium}

The material described by (CR) is isotropic when the following additional symmetry, which we assume throughout this section, is present.

(I) There is a continuous function $\varepsilon:[0, \infty) \rightarrow(0, \infty)$ such that

$$
\varepsilon_{1}\left(s_{1}, s_{2}\right)=\varepsilon_{2}\left(s_{1}, s_{2}\right)=\varepsilon\left(s_{1}+s_{2}\right) \quad \text { for all } s_{1}, s_{2} \geq 0
$$

where $0<A \leq \varepsilon(s)$ for all $s \geq 0$.

In this case the dielectric response is completely determined by the scalar function $\varepsilon$ and so the preceding discussion can be simplified. Furthermore the scalar character of the response is reflected in the equivalent form $\left(\mathrm{CR}^{*}\right)$. To expose this we begin by observing that (I) implies that (V) is satisfied with

$$
\varphi\left(s_{1}, s_{2}\right)=\int_{0}^{s_{1}+s_{2}} \varepsilon(t) d t
$$

and so

$$
\Phi\left(s_{1}, s_{2}\right)=\int_{0}^{\frac{1}{2}|s|^{2}} \varepsilon(t) d t \quad \text { for } s=\left(s_{1}, s_{2}\right) \in \Re^{2}
$$

where $|s|^{2}=s_{1}^{2}+s_{2}^{2}$.

It is now easy to verify that the condition (C) is satisfied by an isotropic medium provided that the function $\varepsilon$ has the following properties.

(A) $\varepsilon \in C^{1}((0, \infty))$ with $\lim _{s \rightarrow 0} s \varepsilon^{\prime}(s)=0$ and $2 s \varepsilon^{\prime}(s)+\varepsilon(s)$ for all $s>0$.

The last inequality (which can be expressed as $\left[\varepsilon\left(\frac{1}{2} s^{2}\right) s\right]^{\prime}$ for all $s>0$ ) ensures that, with $s=\left|\left(s_{1}, s_{2}\right)\right|$,

$$
D^{2} \Phi\left(s_{1}, s_{2}\right)=\left[\begin{array}{cc}
s_{1}^{2} \varepsilon^{\prime}\left(\frac{1}{2} s^{2}\right)+\varepsilon\left(\frac{1}{2} s^{2}\right) & s_{1} s_{2} \varepsilon^{\prime}\left(\frac{1}{2} s^{2}\right) \\
s_{1} s_{2} \varepsilon^{\prime}\left(\frac{1}{2} s^{2}\right) & s_{2}^{2} \varepsilon^{\prime}\left(\frac{1}{2} s^{2}\right)+\varepsilon\left(\frac{1}{2} s^{2}\right)
\end{array}\right]
$$

is positive definite and we note that it is always satisfied if the material is self-focusing since in this case $\varepsilon$ is non-decreasing and $\varepsilon(0)>0$. Furthermore the same inequality implies that

$$
f(s) \equiv \varepsilon\left(\frac{1}{2} s^{2}\right) s
$$

is an increasing, odd diffeomorphism of $\Re$ onto itself. Setting $g=f^{-1}$, we find that

$$
\begin{aligned}
& \Phi\left(s_{1}, s_{2}\right)=\int_{0}^{|s|} f(t) d t \quad \text { for all } s=\left(s_{1}, s_{2}\right) \in \Re^{2} \text { and } \\
& \Phi^{*}\left(\tau_{1}, \tau_{2}\right)=\int_{0}^{|\tau|} g(t) d t \quad \text { for all } \tau=\left(\tau_{1}, \tau_{2}\right) \in \Re^{2} .
\end{aligned}
$$


Thus

$$
\psi\left(\tau_{1}, \tau_{2}\right)=\Gamma\left(\tau_{1}+\tau_{2}\right) \text { for } \tau_{1}, \tau_{2} \geq 0
$$

where $\Gamma(t)=\int_{0}^{\sqrt{2 t}} g(u) d u$.

Setting

$$
\gamma(t)=\Gamma^{\prime}(t) \text { for all } t \geq 0
$$

we have that

$$
g(t)=\gamma\left(\frac{1}{2} t^{2}\right) t \text { for all } t \geq 0
$$

and that

$$
\gamma_{1}\left(\tau_{1}, \tau_{2}\right)=\gamma_{2}\left(\tau_{1}, \tau_{2}\right)=\gamma\left(\tau_{1}+\tau_{2}\right) \text { for } \tau_{1}, \tau_{2} \geq 0
$$

Thus

$$
\gamma\left(\frac{1}{2} t^{2}\right) t=s \Leftrightarrow \varepsilon\left(\frac{1}{2} s^{2}\right) s=t
$$

and

$$
\gamma\left(\frac{1}{2} t^{2}\right)=\frac{1}{\varepsilon\left(\frac{1}{2} s^{2}\right)} \text { where } t=\varepsilon\left(\frac{1}{2} s^{2}\right) s
$$

From (I) and (A) we see that the function $\gamma:[0, \infty) \rightarrow(0, \infty)$ has the following properties which correspond to the condition $(\mathrm{H})$ for the more general uni-axial medium.

(K) $\gamma \in C^{1}((0, \infty)) \cap C([0, \infty))$ with $\gamma(0)=1 / \varepsilon(0), 2 \tau \gamma^{\prime}(\tau)+\gamma(\tau)>0$ for all $\tau>0$ and $\lim _{\tau \rightarrow 0} \tau \gamma^{\prime}(\tau)=0$.

Using the above formulae we find that $\varepsilon$ and $\gamma$ are related by the following identity which can be used to give a more direct definition of $\gamma$,

$$
\varepsilon\left(u \gamma(u)^{2}\right) \gamma(u)=1 \text { for all } u>0 .
$$

Setting $\xi(u, \gamma)=\varepsilon\left(u \gamma^{2}\right) \gamma-1$, it follows from (I) and (A) that

$\xi \in C^{1}((0, \infty) \times \Re)$ and the implicit function theorem defines a function $\gamma \in C^{1}((0, \infty))$ such that $\varepsilon\left(u \gamma(u)^{2}\right) \gamma(u)=1$ for all $u>0$. From this we can recover all of the properties of $\gamma$.

Furthermore additional properties of $\varepsilon$ can easily be translated into information about $\gamma$. We record a few of these implications for future reference. 
1. For $u>0$ and $v=u \gamma(u)^{2}$, we have $u=v \varepsilon(v)^{2}$ and

$$
\frac{d}{d u} \gamma(u)=-\frac{\varepsilon^{\prime}(v)}{\varepsilon(v)^{2}} \frac{d v}{d u}=-\frac{\varepsilon^{\prime}(v)}{\varepsilon(v)^{3}\left\{2 v \varepsilon^{\prime}(v)+\varepsilon(v)\right\}} .
$$

Hence $\gamma^{\prime} \leq 0$ on $(0, \infty) \Leftrightarrow \varepsilon^{\prime} \geq 0$ on $(0, \infty)$.

2. If $\lim _{s \rightarrow \infty} \varepsilon(s)$ exists then $\lim _{\tau \rightarrow \infty} \gamma(\tau)=1 / \lim _{s \rightarrow \infty} \varepsilon(s)$.

3. If there exist $\sigma>0$ and $L \neq 0$ such that

$$
\lim _{s \rightarrow 0} \frac{\varepsilon(s)-\varepsilon(0)}{s^{\sigma}}=L \text { then } \lim _{\tau \rightarrow 0} \frac{\gamma(\tau)-\gamma(0)}{\tau^{\sigma}}=-\frac{L}{\varepsilon(0)^{2(1+\sigma)}}
$$

since

$$
\begin{aligned}
& \frac{\gamma(u)-\gamma(0)}{u^{\sigma}}=\frac{\{\varepsilon(0)-\varepsilon(v)\}}{u^{\sigma} \varepsilon(0) \varepsilon(v)} \\
=- & \frac{\{\varepsilon(v)-\varepsilon(0)\}}{v^{\sigma}} \frac{1}{\varepsilon(0) \varepsilon(v)}\left(\frac{v}{u}\right)^{\sigma}
\end{aligned}
$$

and $\frac{u}{v}=\varepsilon(v)^{2}$.

Finally we remark that in a heterogeneous medium the function $\gamma(x, y, z, u)$ can be defined as the unique positive solution of

$$
\varepsilon\left(x, y, z, u \gamma^{2}\right) \gamma=1 \text { for all } u>0 .
$$

\subsection{Examples}

As a first example of the above procedure let us consider the Kerr law, [4], [17], [37], for a uniaxial material. We suppose that there are four positive constants $\alpha_{T}, \alpha_{z}, P$ and $Q$ such that

$$
\begin{aligned}
& \varepsilon_{1}\left(s_{1}, s_{2}\right)=\alpha_{T}+P s_{1}+Q s_{2} \\
& \varepsilon_{2}\left(s_{1}, s_{2}\right)=\alpha_{z}+Q s_{1}+P s_{2} \text { for all } s_{1}, s_{2} \geq 0 .
\end{aligned}
$$

Clearly $\varepsilon=\nabla \varphi$ where $\varphi\left(s_{1}, s_{2}\right)=\alpha_{T} s_{1}+\alpha_{z} s_{2}+Q s_{1} s_{2}+P\left(s_{1}^{2}+s_{2}^{2}\right) / 2$ and it is easy to check that $\Phi\left(s_{1}, s_{2}\right)=\varphi\left(s_{1}^{2} / 2, s_{2}^{2} / 2\right)$ satisfies the condition (C) provided that $Q / P \leq 3$. Referring to [4] or to page 246 of [8], we see that the ratio $Q / P$ is $\frac{1}{3}$ when the nonlinearity of the dielectric response is due to electronic distortion, whereas it is 1 when this nonlinearity is caused by electrostriction. In a heterogeneous medium $\alpha_{T}, \alpha_{z}, P$ and $Q$ become functions of $(x, y, z)$. 
The medium described by this Kerr law is isotropic provided that $\alpha_{T}=\alpha_{z}$ and $P=Q$. In this case

$$
\varepsilon(s)=\alpha+P s
$$

and so $\gamma(\tau)$ is the unique positive solution of the cubic equation

$$
P \tau \gamma^{3}+\alpha \gamma-1=0
$$

However the Kerr law is at best an approximation which is valid at small to medium field strengths, [31], but which is unacceptable as the field strength becomes infinite since it implies unbounded growth of the dielectric response. In the past decade there has been increasing theoretical and experimental interest in the behaviour of materials under the assumption that the dielectric response remains bounded as the field strength becomes infinite. This property is usually referred to as saturation and for isotropic media two models have attracted considerable attention, [4], [19], [34], [38], [39], namely,

$$
\varepsilon(s)=\alpha+\frac{R P s}{R+P s}
$$

and

$$
\varepsilon(s)=\alpha+R\left[1-e^{-P s / R}\right] .
$$

where $\alpha, P$ and $R$ are positive constants. Both of these responses are approximated by the Kerr law as the field strength tends to zero. Some materials do not behave according to the Kerr law even at small field strengths, [19], $[20],[35]$, and for them models of the following type have been used,

$$
\varepsilon(s)=\alpha+\frac{R P s^{\sigma}}{R+P s^{\sigma}}
$$

where the extra parameter $\sigma$ is also positive. For some semiconductors values of $\sigma$ between 0 and 1 are appropriate. We observe that these three models for isotropic media all satisfy the conditions (I) and (A), and in fact represent self-focusing media since $\varepsilon$ is an increasing function.

Reasonable models for the response of uni-axial materials with saturation can be generated from any isotropic response $\varepsilon$ by setting

$$
\varphi\left(s_{1}, s_{2}\right)=\alpha_{T} s_{1}+\alpha_{z} s_{2}+\int_{0}^{\sqrt{s_{1}^{2}+2 G s_{1} s_{2}+s_{2}^{2}}}\{\varepsilon(s)-\varepsilon(0)\} d s
$$

where $\alpha_{T}, \alpha_{z}$ and $G$ are positive constants. Then the response is defined by

$$
\varepsilon_{i}\left(s_{1}, s_{2}\right)=\partial_{i} \varphi\left(s_{1}, s_{2}\right) \text { for } s_{1}, s_{2} \geq 0 \text {. }
$$


We note that this construction generates the anisotropic Kerr law from the isotropic one by setting $\varepsilon(s)-\varepsilon(0)=P s$ and $G=Q / P$. On the other hand, setting $G=1$ in (14) we see that

$$
\varphi\left(s_{1}, s_{2}\right)=\left[\alpha_{T}-\varepsilon(0)\right] s_{1}+\left[\alpha_{z}-\varepsilon(0)\right] s_{2}+\int_{0}^{s_{1}+s_{2}} \varepsilon(s) \quad d s
$$

and so the response will be isotropic if $G=1$ and $\alpha_{T}=\alpha_{z}$.

Proposition 2 Let the function $\varepsilon$ satisfy the conditions (I) and (A) with $\varepsilon(s) \geq \varepsilon(0)$ for all $s \geq 0$. Then the response defined using the formulae (14) and (15) has the properties (V) and (C) provided that $G \leq 3$ and $\min \left\{\alpha_{T}, \alpha z\right\} \geq \varepsilon(0)$.

Proof. Clearly (V) holds and

$$
\Phi\left(s_{1}, s_{2}\right)=\frac{1}{2}\left\{\alpha_{T} s_{1}^{2}+\alpha_{z} s_{2}^{2}\right\}+\int_{0}^{B\left(s_{1}, s_{2}\right)}\{\varepsilon(s)-\varepsilon(0)\} d s
$$

where

$$
B\left(s_{1}, s_{2}\right)=\frac{1}{2}\left\{s_{1}^{4}+2 G s_{1}^{2} s_{2}^{2}+s_{2}^{4}\right\}^{\frac{1}{2}} .
$$

For $\left(s_{1}, s_{2}\right) \neq(0,0)$,

$$
\begin{aligned}
& D^{2} \Phi\left(s_{1}, s_{2}\right) \\
& =\left[\begin{array}{cc}
\alpha_{T} & 0 \\
0 & \alpha_{z}
\end{array}\right]+\varepsilon^{\prime}\left(B\left(s_{1}, s_{2}\right)\right) B\left(s_{1}, s_{2}\right) \frac{D B\left(s_{1}, s_{2}\right) \otimes D B\left(s_{1}, s_{2}\right)}{B\left(s_{1}, s_{2}\right)} \\
& +\left\{\varepsilon\left(B\left(s_{1}, s_{2}\right)\right)-\varepsilon(0)\right\} D^{2} B\left(s_{1}, s_{2}\right)
\end{aligned}
$$

where

$$
\begin{aligned}
& \frac{D B\left(s_{1}, s_{2}\right) \otimes D B\left(s_{1}, s_{2}\right)}{2 B\left(s_{1}, s_{2}\right)} \\
& =\frac{1}{8 B\left(s_{1}, s_{2}\right)^{3}}\left[\begin{array}{cc}
s_{1}^{2}\left(s_{1}^{2}+G s_{2}^{2}\right)^{2} & s_{1} s_{2}\left(s_{1}^{2}+G s_{2}^{2}\right)\left(G s_{1}^{2}+s_{2}^{2}\right) \\
s_{1} s_{2}\left(s_{1}^{2}+G s_{2}^{2}\right)\left(G s_{1}^{2}+s_{2}^{2}\right) & s_{2}^{2}\left(G s_{1}^{2}+s_{2}^{2}\right)^{2}
\end{array}\right]
\end{aligned}
$$

and

$$
\begin{aligned}
& D^{2} B\left(s_{1}, s_{2}\right) \\
& =\frac{1}{8 B\left(s_{1}, s_{2}\right)^{3}}\left[\begin{array}{cc}
s_{1}^{6}+3 G s_{1}^{4} s_{2}^{2}+3 s_{1}^{2} s_{2}^{4}+G s_{2}^{6} & 2 s_{1}^{3} s_{2}^{3}\left(G^{2}-1\right) \\
2 s_{1}^{3} s_{2}^{3}\left(G^{2}-1\right) & G s_{1}^{6}+3 s_{1}^{4} s_{2}^{2}+3 G s_{1}^{2} s_{2}^{4}+s_{2}^{6}
\end{array}\right]
\end{aligned}
$$


Using (A), we see that $\Phi \in C^{2}\left(\Re^{2}\right)$ with $\Phi(0,0)=\left[\begin{array}{cc}\alpha_{T} & 0 \\ 0 & \alpha_{z}\end{array}\right]$.

Furthermore

$$
\begin{aligned}
& D^{2} \Phi\left(s_{1}, s_{2}\right) \\
& =\left[\begin{array}{cc}
\alpha_{T} & 0 \\
0 & \alpha_{z}
\end{array}\right]-\varepsilon(0) \frac{D B\left(s_{1}, s_{2}\right) \otimes D B\left(s_{1}, s_{2}\right)}{2 B\left(s_{1}, s_{2}\right)} \\
& +\left\{2 \varepsilon^{\prime}\left(B\left(s_{1}, s_{2}\right)\right) B\left(s_{1}, s_{2}\right)+\varepsilon\left(B\left(s_{1}, s_{2}\right)\right)\right\} \frac{D B\left(s_{1}, s_{2}\right) \otimes D B\left(s_{1}, s_{2}\right)}{2 B\left(s_{1}, s_{2}\right)} \\
& +\left\{\varepsilon\left(B\left(s_{1}, s_{2}\right)\right)-\varepsilon(0)\right\}\left\{D^{2} B\left(s_{1}, s_{2}\right)-\frac{D B\left(s_{1}, s_{2}\right) \otimes D B\left(s_{1}, s_{2}\right)}{2 B\left(s_{1}, s_{2}\right)}\right\}
\end{aligned}
$$

where

$$
\begin{aligned}
& D^{2} B\left(s_{1}, s_{2}\right)-\frac{D B\left(s_{1}, s_{2}\right) \otimes D B\left(s_{1}, s_{2}\right)}{2 B\left(s_{1}, s_{2}\right)} \\
& =\frac{\left\{G s_{1}^{4}+\left(3-G^{2}\right) s_{1}^{2} s_{2}^{2}+G s_{2}^{4}\right\}}{8 B\left(s_{1}, s_{2}\right)^{3}}\left[\begin{array}{cc}
s_{2}^{2} & -s_{1} s_{2} \\
-s_{1} s_{2} & s_{2}^{1}
\end{array}\right] .
\end{aligned}
$$

It follows that

$$
\frac{D B\left(s_{1}, s_{2}\right) \otimes D B\left(s_{1}, s_{2}\right)}{2 B\left(s_{1}, s_{2}\right)} \geq 0
$$

and, since $\left\{G s_{1}^{4}+\left(3-G^{2}\right) s_{1}^{2} s_{2}^{2}+G s_{2}^{4}\right\} \geq 0$ for $0<G \leq 3$,

$$
D^{2} B\left(s_{1}, s_{2}\right)-\frac{D B\left(s_{1}, s_{2}\right) \otimes D B\left(s_{1}, s_{2}\right)}{2 B\left(s_{1}, s_{2}\right)} \geq 0 .
$$

Furthermore,

$$
\left[\begin{array}{cc}
\alpha_{T} & 0 \\
0 & \alpha_{z}
\end{array}\right]-\varepsilon(0) \frac{D B\left(s_{1}, s_{2}\right) \otimes D B\left(s_{1}, s_{2}\right)}{2 B\left(s_{1}, s_{2}\right)}
$$

is positive definite for $G \neq 1$ since the Euclidean matrix norm of $M\left(s_{1}, s_{2}\right)=\frac{D B\left(s_{1}, s_{2}\right) \otimes D B\left(s_{1}, s_{2}\right)}{2 B\left(s_{1}, s_{2}\right)}$ is less than 1 . In fact,

$$
\begin{aligned}
\operatorname{det} M\left(s_{1}, s_{2}\right) & =0 \text { and } \\
\operatorname{trace} M\left(s_{1}, s_{2}\right) & =\frac{s_{1}^{6}+3 G s_{1}^{4} s_{2}^{2}+3 G s_{1}^{2} s_{2}^{4}+s_{2}^{6}}{\left\{s_{1}^{4}+2 G s_{1}^{2} s_{2}^{2}+s_{2}^{4}\right\}^{\frac{3}{2}}} .
\end{aligned}
$$


Thus for all $\left(s_{1}, s_{2}\right) \neq(0,0)$,

$$
\begin{aligned}
\left\|M\left(s_{1}, s_{2}\right)\right\| & =\operatorname{trace} M\left(s_{1}, s_{2}\right) \\
& \leq \sup \left\{\frac{1+3 G t+3 G t^{2}+t^{3}}{\left(1+2 G t+t^{2}\right)^{\frac{3}{2}}}: t>0\right\}=\frac{(3 G+1)^{2}}{2(G+1)^{3}} \\
& <1 \text { for } G \geq 0 \text { and } G \neq 1 .
\end{aligned}
$$

For $G=1$, the result follows easily from (16). 


\section{$3 \quad$ Magnetic field wave equations}

For monochromatic waves of the form (1) we show how Maxwell's equations with the constitutive assumption (CR) can be reduced to an equation for the magnetic field alone. This is done in four stages dealing in turn with the general situation, the general equation for TM-modes followed by the special case of TM-modes with planar and cylindrical symmetry. For isotropic media, an earlier version of this discussion was presented in [21]. Now we deal with uni-axial anisotropy, assuming throughout a constitutive relation of the form (CR) in which the dielectric the response has the properties (V) and (C).

Starting with an arbitrary (sufficiently smooth) field $H$ in the form (1), we define the fields $B, D$ and $E$ as follows.

$$
B=H
$$

then

$$
\begin{aligned}
D(x, y, z, t) & =-\frac{c}{\omega^{2}} \nabla \wedge \partial_{t} H(x, y, z, t) \\
& =-\frac{c}{\omega} \nabla \wedge\left\{H_{T} \sin (k z-\omega t)-H_{z} \cos (k z-\omega t)\right\}
\end{aligned}
$$

and finally

$$
E(x, y, z, t)=E_{T}(x, y) \cos (k z-\omega t)+E_{z}(x, y) \sin (k z-\omega t)
$$

where

$$
\begin{aligned}
E_{T}(x, y) & =\gamma_{1}\left(\left|D_{T}(x, y)\right|^{2} / 2,\left|D_{z}(x, y)\right|^{2} / 2\right) D_{T}(x, y) \\
E_{z}(x, y) & =\gamma_{2}\left(\left|D_{T}(x, y)\right|^{2} / 2,\left|D_{z}(x, y)\right|^{2} / 2\right) D_{z}(x, y) .
\end{aligned}
$$

It follows from these definitions that the equations $\left(\mathrm{ME}_{2}\right)$ and $\left(\mathrm{ME}_{3}\right)$ are satisfied as is $\left(\mathrm{CR}^{*}\right)$ and hence, by the properties of $\gamma_{i}$, so is $(\mathrm{CR})$. Thus it is enough to choose $H$ in such a way that the equations $\left(\mathrm{ME}_{1}\right)$ and $\left(\mathrm{ME}_{4}\right)$ hold. Before doing this it is convenient to introduce some notation which enables us to express $D$ and $E$ explicitly in terms of $H$. Let

$$
\begin{aligned}
H_{T}(x, y) & =\frac{\omega}{c}\left\{h_{1}(x, y) e_{1}+h_{2}(x, y) e_{2}\right\} \\
\text { and } \quad H_{z}(x, y) & =\frac{\omega}{c} h_{3}(x, y) e_{3} .
\end{aligned}
$$


where $h_{i} \in C^{2}\left(\Re^{2}\right)$ for $i=1,2,3$. Then

$$
D(x, y, z, t)=D_{T}(x, y) \cos (k z-\omega t)+D_{z}(x, y) \sin (k z-\omega t)
$$

where

$$
\begin{aligned}
D_{T}(x, y) & =\left[\partial_{y} h_{3}+k h_{2}\right] e_{1}-\left[\partial_{x} h_{3}+k h_{1}\right] e_{2} \\
D_{z}(x, y) & =-\left[\partial_{x} h_{2}-\partial_{y} h_{1}\right] e_{3}
\end{aligned}
$$

so that

$$
\begin{aligned}
\left|D_{T}(x, y)\right|^{2} & =\left[\partial_{y} h_{3}+k h_{2}\right]^{2}+\left[\partial_{x} h_{3}+k h_{1}\right]^{2} \\
\left|D_{z}(x, y)\right|^{2} & =\left[\partial_{x} h_{2}-\partial_{y} h_{1}\right]^{2}
\end{aligned}
$$

and

$$
\begin{aligned}
E_{T}(x, y) & =\widetilde{\gamma}_{1}(x, y)\left\{\left[\partial_{y} h_{3}+k h_{2}\right] e_{1}-\left[\partial_{x} h_{3}+k h_{1}\right] e_{2}\right\} \\
E_{z}(x, y) & =\widetilde{\gamma}_{2}(x, y)\left[-\partial_{x} h_{2}+\partial_{y} h_{1}\right] e_{3} .
\end{aligned}
$$

where

$$
\widetilde{\gamma}_{i}(x, y)=\gamma_{i}\left(\left\{\left[\partial_{y} h_{3}+k h_{2}\right]^{2}+\left[\partial_{x} h_{3}+k h_{1}\right]^{2}\right\} / 2,\left[\partial_{x} h_{2}-\partial_{y} h_{1}\right]^{2} / 2\right) .
$$

Thus the equation $\left(\mathrm{ME}_{1}\right)$ is satisfied provided that

$$
\begin{aligned}
& \nabla \wedge\left\{E_{T}(x, y) \cos (k z-\omega t)+E_{z}(x, y) \sin (k z-\omega t)\right\} \\
& =-\frac{\omega}{c}\left\{H_{T} \sin (k z-\omega t)-H_{z} \cos (k z-\omega t)\right\}
\end{aligned}
$$

and, if $H$ is a solution of this equation, then $\left(\mathrm{ME}_{4}\right)$ is automatically satisfied and the above construction furnishes a solution of (ME) with (CR). Using the expression for $E$ in terms of $H$ the fundamental equation for the magnetic field becomes

$$
\begin{aligned}
\partial_{y}\left\{\tilde{\gamma}_{2}\left[\partial_{x} h_{2}-\partial_{y} h_{1}\right]\right\}+k \tilde{\gamma}_{1}\left[\partial_{x} h_{3}+k h_{1}\right] & =\left(\frac{\omega}{c}\right)^{2} h_{1} \\
-\partial_{x}\left\{\tilde{\gamma}_{2}\left[\partial_{x} h_{2}-\partial_{y} h_{1}\right]\right\}+k \tilde{\gamma}_{1}\left[\partial_{y} h_{3}+k h_{1}\right] & =\left(\frac{\omega}{c}\right)^{2} h_{2} \\
-\partial_{x}\left\{\tilde{\gamma}_{1}\left[\partial_{x} h_{3}+k h_{1}\right]\right\}-\partial_{y}\left\{\tilde{\gamma}_{1}\left[\partial_{y} h_{3}+k h_{1}\right]\right\} & =\left(\frac{\omega}{c}\right)^{2} h_{3}
\end{aligned}
$$


or it can be expressed equivalently as

$$
\begin{aligned}
\partial_{y}\left\{\tilde{\gamma}_{2}\left[\partial_{x} h_{2}-\partial_{y} h_{1}\right]\right\}+k \tilde{\gamma}_{1}\left[\partial_{x} h_{3}+k h_{1}\right] & =\left(\frac{\omega}{c}\right)^{2} h_{1} \\
-\partial_{x}\left\{\tilde{\gamma}_{2}\left[\partial_{x} h_{2}-\partial_{y} h_{1}\right]\right\}+k \tilde{\gamma}_{1}\left[\partial_{y} h_{3}+k h_{1}\right] & =\left(\frac{\omega}{c}\right)^{2} h_{2} \\
\partial_{x} h_{1}+\partial_{y} h_{2}+k h_{3} & =0 .
\end{aligned}
$$

In this way, (ME) with (CR) are reduced to a system of three equations for the three unknowns $h_{i}$ which are functions of $(x, y)$. Indeed $h_{3}$ can immediately be eliminated so as to leave a system of two equations for $h_{1}$ and $h_{2}$. However, on recalling the definition of the functions $\tilde{\gamma}_{i}$, we see that the system is highly nonlinear.

\subsection{General TM-modes}

The formulation above is particularly attractive when the solutions which are being sought are such that the magnetic field has a simpler form than the electric field. Here we discuss solutions having the form (1) with

$$
H_{z}(x, y) \equiv 0
$$

These solutions are called TM-modes since the condition (19) means that the magnetic field is transverse to the direction of propagation of the wave. For these modes, $h_{3} \equiv 0$, and the system (18) becomes

$$
\begin{aligned}
\partial_{y}\left\{\tilde{\gamma}_{2}\left[\partial_{x} h_{2}-\partial_{y} h_{1}\right]\right\}+k^{2} \tilde{\gamma}_{1} h_{1} & =\left(\frac{\omega}{c}\right)^{2} h_{1} \\
-\partial_{x}\left\{\tilde{\gamma}_{2}\left[\partial_{x} h_{2}-\partial_{y} h_{1}\right]\right\}+k^{2} \tilde{\gamma}_{1} h_{1} & =\left(\frac{\omega}{c}\right)^{2} h_{2} \\
\partial_{x} h_{1}+\partial_{y} h_{2} & =0
\end{aligned}
$$

where now

$$
\widetilde{\gamma}_{i}(x, y)=\gamma_{i}\left(k^{2}\left[h_{1}^{2}+h_{2}^{2}\right] / 2,\left[\partial_{x} h_{2}-\partial_{y} h_{1}\right]^{2} / 2\right) .
$$

In seeking TM-modes we must solve a system of three equations for the two unknown functions $h_{1}(x, y)$ and $h_{2}(x, y)$. The next two sections deal with situations where this is possible. 


\subsection{Planar TM-modes}

The simplest case concerns TM-modes having the following special form,

$$
h_{1}(x, y) \equiv 0 \text { and } h_{2}(x, y)=u(k x) / k
$$

Indeed most of the literature on guided TM-modes is devoted to this situation. Using the above formulation, the problem is now reduced to finding $u \in C^{2}(\Re)$ such that

$$
\left\{g_{2}(x) u^{\prime}(x)\right\}^{\prime}-g_{1}(x) u(x)+\lambda u(x)=0
$$

where

$$
\lambda=\left(\frac{\omega}{k c}\right)^{2} \text { and } g_{i}(x)=\gamma_{i}\left(u(x)^{2} / 2, u^{\prime}(x)^{2} / 2\right) .
$$

In this case the e-m fields obtained from a solution of (22) are

$$
\begin{aligned}
& H(x, y, z, t)=B(x, y, z, t)=\left(\frac{\omega}{k c}\right) u(k x) \cos (k z-\omega t) \quad e_{2} \\
& D(x, y, z, t)=u(k x) \cos (k z-\omega t) e_{2}-u^{\prime}(k x) \sin (k z-\omega t) e_{3} \\
& E(x, y, z, t)=g_{1}(k x) u(k x) \cos (k z-\omega t) e_{2}-g_{2}(k x) u^{\prime}(k x) \sin (k z-\omega t) e_{3} .
\end{aligned}
$$

Note that the equation (22) can be written as

$$
\left\{\partial_{2} \Phi^{*}\left(u(r), u^{\prime}(r)\right)\right\}^{\prime}-\partial_{1} \Phi^{*}\left(u(r), u^{\prime}(r)\right)+\lambda u(r)=0 .
$$

\subsection{Cylindrical TM-modes}

Another family of TM-modes can be found by seeking solutions in the form

$$
h_{1}(x, y)=-\left(\frac{u(k r)}{k}\right) \sin \theta \text { and } h_{2}(x, y)=\left(\frac{u(k r)}{k}\right) \cos \theta
$$

where $(r, \theta)$ are the usual polar coordinates in $\Re^{2}$. Modes of this type have a cylindrical symmetry appropriate for optical fibres and, in the electric field formulation, they have been studied numerically in [9], [24]. Using the formulation introduced above the problem is reduced to finding a single function $u \in C^{2}((0, \infty))$ such that

$$
\left\{g_{2}(r)\left[u^{\prime}(r)+\frac{u(r)}{r}\right]\right\}^{\prime}-g_{1}(r) u(r)+\lambda u(r)=0
$$


where

$$
\lambda=\left(\frac{\omega}{k c}\right)^{2} \text { and } g_{i}(r)=\gamma_{i}\left(u(r)^{2} / 2,\left[u^{\prime}(r)+\frac{u(r)}{r}\right]^{2} / 2\right)
$$

In this case the e-m fields obtained from a solution of (25) are

$$
\begin{aligned}
H(x, y, z, t) & =B(x, y, z, t)=\left(\frac{\omega}{k c}\right) u(k r) \cos (k z-\omega t) i_{\theta} \\
D(x, y, z, t) & =u(k r) \cos (k z-\omega t) i_{r}-\left[u^{\prime}(k r)+\frac{u(k r)}{k r}\right] \sin (k z-\omega t) i_{z} \\
E(x, y, z, t) & =g_{1}(k r) u(k r) \cos (k z-\omega t) i_{r} \\
& -g_{2}(k r)\left[u^{\prime}(k r)+\frac{u(k r)}{k r}\right] \sin (k z-\omega t) i_{z}
\end{aligned}
$$

where

$$
i_{r}=\cos \theta e_{1}+\sin \theta e_{2}, i_{\theta}=-\sin \theta e_{1}+\cos \theta e_{2}, i_{z}=e_{3} .
$$

Note that the equation (25) can also be written as

$$
\left\{\partial_{2} \Phi^{*}\left(u(r), u^{\prime}(r)+\frac{u(r)}{r}\right)\right\}^{\prime}-\partial_{1} \Phi^{*}\left(u(r), u^{\prime}(r)+\frac{u(r)}{r}\right)+\lambda u(r)=0 .
$$

Of course these definitions hold for $r>0$ but it is easy so check that the fields can be smoothly extended onto the axis $r=0$ provided that $u$ has the following properties.

$$
\lim _{r \rightarrow 0} u(r)=\lim _{r \rightarrow 0}\left[u^{\prime}(r)+\frac{u(r)}{r}\right]^{\prime}=0
$$

and both $\lim _{r \rightarrow 0} u^{\prime}(r)$ and $\lim _{r \rightarrow 0}\left[u^{\prime}(r)+\frac{u(r)}{r}\right]$ exist and are finite.

However for a solution of (25) these properties are not independent and, as the following result shows, it is sufficient to impose a seemingly weaker condition.

Proposition 3 Let $u \in C^{2}((0, \infty))$ be a solution of (25) such that $\lim _{r \rightarrow 0} u(r)=$ 0 and $u^{\prime}$ is bounded on $(0,1)$. Then $u$ has the properties (26) and (27).

Proof. Setting $u(0)=0$, we have that $u \in C([0, \infty))$. Let

$$
v(r)=u^{\prime}(r)+\frac{u(r)}{r}
$$


and

$$
\alpha=\sup \left\{\left|u^{\prime}(r)\right|: 0<r<1\right\} .
$$

Then, for $0<R<r<1$,

$$
|u(r)-u(R)|=\left|\int_{R}^{r} u^{\prime}(t) d t\right| \leq \alpha(r-R)
$$

and, letting $R \rightarrow 0$, we obtain

$$
\left|\frac{u(r)}{r}\right| \leq \alpha \quad \text { for } \quad 0<r<1 .
$$

This implies that $|v(r)| \leq 2 \alpha$ for $0<r<1$. For $0<r<1$, the equation (25) can be written as

$$
\left\{\partial_{2} \Phi^{*}(u(r), v(r))\right\}^{\prime}-\partial_{1} \Phi^{*}(u(r), v(r))+\lambda u(r)=0
$$

and hence

$$
\partial_{2}^{2} \Phi^{*}(u(r), v(r)) v^{\prime}(r)=h(r)
$$

where

$$
\begin{aligned}
h(r) & =\partial_{1} \Phi^{*}(u(r), v(r))-\lambda u(r)-u^{\prime}(r) \partial_{1} \partial_{2} \Phi^{*}(u(r), v(r)) \\
& =u(r)\left\{\gamma_{1}\left(\frac{u(r)^{2}}{2}, \frac{v(r)^{2}}{2}\right)-\lambda-u^{\prime}(r) v(r) \partial_{1} \gamma_{2}\left(\frac{u(r)^{2}}{2}, \frac{v(r)^{2}}{2}\right)\right\} .
\end{aligned}
$$

Setting

$$
w(r)=\left(\frac{u(r)^{2}}{2}, \frac{v(r)^{2}}{2}\right)
$$

there exists $\beta>0$ such that $|w(r)| \leq \beta$ for all $r \in(0,1)$. Recalling the properties of $\Phi^{*}$, we see that there exist constants $L$ and $K$ such that

$$
\partial_{2}^{2} \Phi^{*}(u(r), v(r)) \geq L>0 \text { and }|h(r)| \leq K \text { for } 0<r<1 .
$$

Thus $\left|v^{\prime}(r)\right| \leq K / L$ for all $r \in(0,1)$ which ensures that $\lim _{r \rightarrow 0} v(r)$ exists and is finite. We set $v(0)=\lim _{r \rightarrow 0} v(r)$. Now by l'Hospital's rule,

$$
\lim _{r \rightarrow 0} \frac{r u(r)}{r^{2}}=\lim _{r \rightarrow 0} \frac{(r u(r))^{\prime}}{2 r}=\frac{1}{2} \lim _{r \rightarrow 0} v(r)=\frac{1}{2} v(0)
$$


and so $\lim _{r \rightarrow 0} \frac{u(r)}{r}$ exists and is equal to $\frac{1}{2} v(0)$.

Since $u^{\prime}(r)=v(r)-\frac{u(r)}{r}$ for $r>0$, we also have that $\lim _{r \rightarrow 0} u^{\prime}(r)$ exists and is equal to $\frac{1}{2} v(0)$. Returning to the differential equation we now see that

$$
\lim _{r \rightarrow 0} v^{\prime}(r)=-\frac{v(0)}{2} \partial_{1} \partial_{2} \Phi^{*}(0, v(0)) / \partial_{2}^{2} \Phi^{*}(0, v(0))
$$

But $\partial_{1} \Phi^{*}\left(0, t_{2}\right)=0$ for all $t_{2}$ and so $\partial_{1} \partial_{2} \Phi^{*}(0, v(0))=0$, proving that $\lim _{r \rightarrow 0} v^{\prime}(r)=0$. 


\section{Existence of guided TM-modes}

The guidance of light is usually achieved by exploiting the effect of variations in the refractive index due to inhomogeneity of the medium through which the beam is propagating, [40]. As can be understood from Snell's law, the favourable configuration consists of a region of high refractive index surrounded by layers of material having a lower refractive index. However it is also well-known that nonlinear effects can be used to enhance, or even to produce, guidance which in this case is often referred to as self-trapping since it is the light beam itself which induces the variation in refractive index keeping the light confined to a region near the axis of propagation, [15], [36]. For example, no guidance occurs in a homogeneous linear medium (where the refractive index is constant) whereas it will occur, at least for sufficiently intense beams, in a homogeneous, isotropic, self-focusing medium (where the refractive index is an increasing function of the intensity of the light passing through it). See [14], [22], [23], [25] for such results concerning TE-modes.

In this section we review some more recent work concerning the existence of guided TM-modes, [2], [3]. We assume throughout a constitutive relation of the form (CR) where the conditions (V) and (C) are satisfied. For planar and then cylindrical symmetry, the results establish additional properties of the dielectric tensor which are sufficient to ensure the existence of guided TM-modes within certain ranges of the wavelength.

\subsection{Guided planar TM-modes}

By a guided planar TM-mode we mean a solution $u \not \equiv 0$ of equation (22) which has the properties that

$$
u \in H^{1}(\Re) \text { and } \lim _{x \rightarrow \pm \infty} u(x)=\lim _{x \rightarrow \pm \infty} u^{\prime}(x)=0
$$

where $H^{1}(\Re)$ is the usual Sobolev space. In this section we discuss the existence of such solutions. The analysis is based on the observation that the function $I$ defined by

$$
I(p, q)=\Phi^{*}(p, q)-q \partial_{2} \Phi^{*}(p, q)-\lambda p^{2} / 2
$$

is a first integral for (22). Indeed, recalling that $\nabla \psi=\gamma=\left(\gamma_{1}, \gamma_{2}\right)$, we see that

$$
I(p, q)=\psi\left(p^{2} / 2, q^{2} / 2\right)-\gamma_{2}\left(p^{2} / 2, q^{2} / 2\right) q^{2}-\lambda p^{2} / 2
$$


and hence it is easy to verify that if $u$ satisfies (22) then

$$
\left\{I\left(u(x), u^{\prime}(x)\right)\right\}^{\prime}=u^{\prime}(x)\left[g_{1} u(x)-\lambda u(x)-\left\{g_{2} u^{\prime}(x)\right\}^{\prime}\right]=0 .
$$

Since $\Phi^{*}(0,0)=0$ and $0<\gamma_{2}(p, q)<1 / A$, it follows that if $u$ satisfies $(22)$ and $(28)$ then

$$
I\left(u(x), u^{\prime}(x)\right)=0 \text { for all } x \in \Re .
$$

Hence the orbit of a guided TM-mode lies in the set $I^{-1}(0) \backslash\{(0,0)\}$ and, in view of the symmetries of $I$, it is sufficient to discuss the set

$$
C=\{(p, q): I(p, q)=0 \text { with } p \geq 0 \text { and } q \geq 0\} .
$$

For this we introduce some additional hypotheses about the dielectric response.

(S) (a) $\varepsilon_{1}\left(s_{1}, 0\right)$ is a strictly increasing function of $s_{1}$ on $[0, \infty)$, and

(b) there exist $B>0$ and $\alpha \geq 0$ such that $\varepsilon_{2}\left(s_{1}, s_{2}\right) / s_{2}^{\alpha} \longrightarrow B$ as $s_{2} \longrightarrow \infty$, uniformly for $s_{1}$ in bounded subsets of $[0, \infty)$.

We observe that, in the example of a Kerr material which was discussed in Section 2.3, the condition (S) is satisfied with $\alpha=1$. On the other hand for realistic constitutive laws which model saturation, the value $\alpha=0$ in $(\mathrm{S})(\mathrm{b})$ is appropriate.

As we showed in Section 2.2, these properties of $\varepsilon_{i}$ imply some analogous behaviour of the functions $\gamma_{i}$. In particular, $\lim _{s_{1} \rightarrow \infty} \varepsilon_{1}\left(s_{1}, 0\right)$ exists and, setting

$$
\varepsilon_{1}(\infty, 0)=\lim _{s_{1} \rightarrow \infty} \varepsilon_{1}\left(s_{1}, 0\right)
$$

we have that

$$
0 \leq \gamma_{1}(\infty, 0)=1 / \varepsilon_{1}(\infty, 0)
$$

where $\gamma_{1}(\infty, 0)=\lim _{t_{1} \rightarrow \infty} \gamma_{1}\left(t_{1}, 0\right)$.

These properties of $\varepsilon_{i}$ can be used to establish the following results [2].

Lemma 4 Let the dielectric response have the properties $(C R),(V),(C)$ and (S).

If $\lambda \notin\left(\gamma_{1}(\infty, 0), \gamma_{1}(0,0)\right)$, then $C \cap\{(p, 0): p>0\}=\emptyset$.

If $\lambda \in\left(\gamma_{1}(\infty, 0), \gamma_{1}(0,0)\right)$, then there exist $p_{\lambda}>0$ and $f \in C\left(\left[0, p_{\lambda}\right]\right) \cap$ $C^{1}\left(\left(0, p_{\lambda}\right)\right)$ such that

$f(0)=0, f\left(p_{\lambda}\right)=0, f(p)>0$ for all $p \in\left(0, p_{\lambda}\right)$

and $C=\left\{(p, f(p)): 0 \leq p \leq p_{\lambda}\right\}$.

Furthermore $\lim _{p \rightarrow 0} f^{\prime}(p)=\sqrt{\frac{\gamma_{1}(0,0)-\lambda}{\gamma_{2}(0,0)}}$ and $\lim _{p \rightarrow p_{\lambda}} f^{\prime}(p)=-\infty$. 
From these properties of $C$ we can deduce the following information about guided TM-modes.

Recall that $\gamma_{1}(0,0)=1 / \varepsilon_{1}(0,0)$ and that $\gamma_{1}(\infty, 0)=1 / \varepsilon_{1}(\infty, 0)$. By a guided planar TM-mode we mean a solution of (22) which satisfies (28).

Theorem 5 Let the dielectric response have the properties $(C R),(V),(C)$ and $(S)$.

If $\lambda \notin\left(\gamma_{1}(\infty, 0), \gamma_{1}(0,0)\right)$, then there is no guided planar TM-mode.

If $\lambda \in\left(\gamma_{1}(\infty, 0), \gamma_{1}(0,0)\right)$, then there exists a unique planar guided TMmode $u_{\lambda}$ such that $u_{\lambda}(0)=p_{\lambda}$ and $u_{\lambda}^{\prime}(0)=0$.

Furthermore, $u_{\lambda}(x)=u_{\lambda}(-x)>0$ for all $x \in \Re$ and,

for any $\mu<\sqrt{\frac{\gamma_{1}(0,0)-\lambda}{\gamma_{2}(0,0)}}, \lim _{x \rightarrow \infty} \exp (\mu x) u_{\lambda}(x)=0$.

All guided TM-modes are of the form $\pm_{\lambda}(x+\delta)$ for some $\delta \in \Re$ and some $\lambda \in\left(\gamma_{1}(\infty, 0), \gamma_{1}(0,0)\right)$.

\subsection{Guided cylindrical TM-modes}

By a guided cylindrical TM-mode we mean a solution $u \not \equiv 0$ of equation (25) which satisfies the regularity conditions (26) and (27) together with the following guidance conditions,

$$
\int_{0}^{\infty}\left\{u(r)^{2}+v(r)^{2}\right\} \quad r d r<\infty
$$

and

$$
\lim _{r \rightarrow \infty} u(r)=\lim _{r \rightarrow \infty} v(r)=0
$$

where $v(r)=u^{\prime}(r)+\frac{u(r)}{r}$. For general modes of the form (1), the power, $P$, of the associated beam of light is defined to be the time-average of the energy flux,

$$
\iint_{\Re^{2}} c(E \wedge H) \cdot e_{3} d x d y,
$$

across a plane perpendicular to the direction of propagation. In the case of cylindrical TM-modes we obtain the expression

$$
\begin{aligned}
P & =\frac{\pi \omega}{k} \int_{0}^{\infty} g_{1}(k r) u(k r)^{2} \quad r d r \\
& =\frac{\pi \omega}{k^{3}} \int_{0}^{\infty} \gamma_{1}\left(u(r)^{2} / 2, v(r)^{2} / 2\right) u(r)^{2} \quad r d r .
\end{aligned}
$$


Henceforth we restrict our attention to the case of an isotropic selffocusing medium and we summarize the main results obtained in [3]. (The approach could be extended to deal with some uni-axial materials.) Thus we replace the conditions $(\mathrm{V})$ and $(\mathrm{C})$ by $(\mathrm{I})$ and $(\mathrm{A})$ and we assume in addition that

(T) $\varepsilon^{\prime} \geq 0$ on $(0, \infty), \varepsilon(\infty) \equiv \lim _{s \rightarrow \infty} \varepsilon(s)<\infty$ and there exist positive constants $\sigma$ and $L$ such that

$$
\lim _{s \rightarrow 0} \frac{\varepsilon(s)-\varepsilon(0)}{s^{\sigma}}=L .
$$

These conditions mean that the material is self-focusing and that the dielectric response saturates as the field strength becomes infinite. As is shown in Section 2.2, they imply that, in addition to $(\mathrm{K})$, the function $\gamma$ has the following properties.

$$
\begin{gathered}
\text { (G) } \gamma^{\prime} \leq 0 \text { on }(0, \infty), \gamma(\infty) \equiv \lim _{t \rightarrow \infty} \gamma(t)>0 \text { and } \\
\lim _{t \rightarrow 0} \frac{\gamma(t)-\gamma(0)}{t^{\sigma}}=-K .
\end{gathered}
$$

where $K=-L / \varepsilon(0)^{2(1+\sigma)}$.

The equation (25) can now be written as

$$
\left\{\gamma\left(\frac{1}{2}\left[u(r)^{2}+v(r)^{2}\right]\right) v(r)\right\}^{\prime}-\gamma\left(\frac{1}{2}\left[u(r)^{2}+v(r)^{2}\right]\right) u(r)+\lambda u(r)=0 .
$$

It turns out that the boundary conditions can be treated in a very convenient way by expressing the problem in terms of the new variable,

$$
z(r)=\sqrt{r} u(r)
$$

In fact, using $z$, guided cylindrical TM-modes are characterized by the following variational principle. On the Sobolev space

$$
H_{0}^{1}(0, \infty)=\left\{z \in L^{2}(0, \infty): z^{\prime} \in L^{2}(0, \infty) \text { and } z(0)=0\right\}
$$

we can define a $C^{1}$-functional by

$$
\begin{aligned}
J(z) & =\int_{0}^{\infty} \Gamma\left(\frac{1}{2 r}\left\{z(r)^{2}+\left[z^{\prime}(r)+\frac{z(r)}{2 r}\right]^{2}\right\}\right) r d r \\
& -\frac{\gamma(0)}{2} \int_{0}^{\infty} z(r)^{2} r d r .
\end{aligned}
$$


where $\Gamma(t)=\int_{0}^{\infty} \gamma(\tau) d \tau$. For $d>0$, we set

$$
S(d)=\left\{z \in H_{0}^{1}(0, \infty):\|z\|=d\right\}
$$

where $\|z\|^{2}=\int_{0}^{\infty} z(r)^{2} d r$. Every stationary point, $z$, of $J$ restricted to $S(d)$ satisfies the Euler-Lagrange equation

$$
J^{\prime}(z) \varphi=\xi \int_{0}^{\infty} z(r) \varphi(r) d r \text { for all } \varphi \in H_{0}^{1}(0, \infty)
$$

and as the next result asserts they correspond precisely to the solutions we are seeking.

Theorem 6 Let the dielectric response have the properties (CR), (I), (A) and $(T)$.

(i) $J \in C^{1}\left(H_{0}^{1}(0, \infty)\right)$.

(ii) Let $(\xi, z) \in \Re \times H_{0}^{1}(0, \infty)$ satisfy (36) where $z \not \equiv 0$ and set $\lambda=$ $\xi+\gamma(0), u(r)=z(r) / \sqrt{r}$. Then $\lambda>\gamma(\infty)$ and $(\lambda, u)$ is a guided cylindrical TM-mode. Conversely, if $(\lambda, u)$ is a guided cylindrical TM-mode then $\lambda>$ $\gamma(\infty), z \in H_{0}^{1}(0, \infty)$ and (36) is satisfied with $\xi=\lambda-\gamma(0)$.

(ii) If $(\lambda, u)$ is a guided cylindrical TM-mode with $\lambda<\gamma(0)$ then $u$ and $v$ decay exponentially as $r \rightarrow \infty$.

The existence of guided cylindrical TM-modes is thus reduced to the search for stationary points of $\left.J\right|_{S(d)}$ for $d>0$. Since $J$ is bounded below on $S(d)$, we can begin by trying to show that $J$ attains its minimum on $S(d)$. However, as the next results show, although this is the case for all sufficiently large values of $d$, it is false for small values of $d$ unless the exponent $\sigma$ in (T) is less than 1.

Theorem 7 Let the dielectric response have the properties (CR), (I), (A) and $(T)$. For $d>0$, set

$$
m(d)=\inf \{J(z): z \in S(d)\} .
$$

(i) Then $m(d) \geq-\frac{1}{2}[\gamma(0)-\gamma(\infty)] d^{2}$ and there exists $d_{0} \geq 0$ such that, for all $d>d_{0}, m(d)<0$ and there exists $z_{d} \in S(d)$ such that $J\left(z_{d}\right)=m(d)$. Furthermore, $z_{d}>0$ on $(0, \infty)$ and there exists $\lambda_{d}$ such that $\left(\lambda_{d}, u_{d}\right)$ is a guided cylindrical TM-mode where $u_{d}(r)=z_{d}(r) / \sqrt{r}$ and $\gamma(\infty)<\lambda_{d}<$ $\gamma(0)+2 m(d) / d^{2}<\gamma(0)$. Also $\lim _{d \rightarrow \infty} \lambda_{d}=\gamma(\infty)$ and $\lim _{d \rightarrow \infty} P_{d}=\infty$ where $P_{d}$ is the power of the beam associated with $\left(\lambda_{d}, u_{d}\right)$.

(ii) If $\sigma \in(0,1)$ we may choose $d_{0}=0$ and in this case $\lim _{d \rightarrow 0} \lambda_{d}=\gamma(0)$ and $\lim _{d \rightarrow 0} P_{d}=0$.

(iii) If $\sigma \geq 1$, then $\lambda \leq \gamma(0)$ and there exists a constant $d_{1}>0$ such that $\|u\| \geq d_{1}$ for every guided cylindrical TM-mode $(\lambda, u)$. The power of such modes is also bounded away from zero. 
Under slightly more restrictive assumptions on the dielectric response Ruppen, [41], as established the existence of non-positive guided cylindrical TM-modes by showing that there are stationary points of $\left.J\right|_{S(d)}$ which are not minima. 


\section{References}

\section{References}

[1] Born, M. and Wolf, E. : Principles of Optics, fifth edition, Pergamon Press, Oxford, 1975.

[2] Stuart, C. A. : Guided TM-modes in a self-focusing anisotropic dielectric, in Nonlinear Problems in Applied Mathematics, edited by T. S. Angell et al., SIAM Proceedings Series, Philadelpdia, 1995.

[3] Stuart, C. A. : Cylindrical TM-modes in a homogeneous self-focusing dielectric, to appear in Math. Models \& Methods Appl. Sci. 1996.

[4] Mihalache, D., Bertolotti, M. and Sibilia, C. : Nonlinear wave propagation in planar structures, in Progress in Optics XXVII, edited by E. Wolf, Elsevier, 1987.

[5] Akmediev, N. N. : Nonlinear theory of surface polaritons, Sov. Phys., JETP. 57 (1983), 1111-1116.

[6] Mihalache, D., Stegeman, G. I., Seaton, C. T., Wright, E. M., Zanoni, R., Boardman, A. D. and Twardowski, T. : Exact dispersion relations for transverse magnetic polarized guided waves at a non-linear interface, Opt. Lett. 12 (1987), 187-189.

[7] Joseph, R. I. and Christodoulides, D. N. : Exact field decomposition for TM waves in nonlinear media, Opt. Lett. 10 (1987), 826-828.

[8] Ogusu, K. : TM waves guided by nonlinear planar waveguides, IEEE, Trans. Microwave Th. et Tech., 37 (1989), 941-946.

[9] Chen, Y. : TE and TM families of self-trapped beams, IEEE J. Quantum Elect., 27 (1991), 1236-1241.

[10] Yokota, M. : Guided transverse-magnetic waves supported by a weakly nonlinear slab waveguide, J. Opt. Soc. Am. B10 (1993), 1096-1101.

[11] Wang, X. H. and Cambrell, G. K. : Full vectorial simulation of bistability phenomena in nonlinear-optical channel wave-guides, J. Opt. Soc. Am. B10 (1993), 1090-1095. 
[12] Wang, X. H. and Cambrell, G. K. : Simulation of strong nonlinear effects in optical waveguides, J. Opt. Soc.Am. B11 (1993), 2048-2055.

[13] Leung, K. M. : p-polarized nonlinear surface polaritons in materials with intensity-dependent dielectric function, Phy. Rev. B32 (1985), 50935101.

[14] Stuart, C. A. : Self-trapping of an electromagnetic field and bifurcation from the essential spectrum, Arch. Rational ;Mech. Anal., 113 (1991), 65-96.

[15] Stegeman, G. I. : Nonlinear guided wave optics, in Contemporary nonlinear optics, Edited by G. P. Agrawal and R. W. Boyd, Academic Press, Boston, 1992.

[16] Akhmanov, R.-V., Khorklov, R. V. \& Sukhorukov, A. P. : Self-focusing, self-defocusing and self-modulation of laser beams, in Laser Handbook, edited by F.T. Arecchi \& E.O. Schulz Dubois, North-Holland, Amsterdam, 1972.

[17] Svelto, O. : Self-focusing, self-trapping and self-phase modulation of laser beams, in Progress in Optics, Vol. 12, editor E. Wolf, NorthHolland, Amsterdam, 1974.

[18] Reintjes, J. F. : Nonlinear Optical Processes, in Encyclopedia of Physical Science and Technology, Vol. 9, Academic Press, New York.

[19] Stegeman, G. I., Ariyant, J., Seaton, C. I., Shen, T.-P. \& Moloney, J. V. : Nonlinear thin-film guided waves in non-Kerr media, Appl. Phys. Lett. 47 (1985), 1254-1256.

[20] Mathew, J. G. H., Lar, A. K., Heckenberg, N. R. \& Galbraith, I. : Time resolved self-defocusing in InSb at room temperature, IEEE J. Quantum Elect. 21 (1985), 94-99.

[21] Stuart, C. A. : Magnetic field wave equation for nonlinear optical waveguides, Rapport Nr 11.93, Départ. Math., Ecole Polytechnique Fédérale de Lausanne, 1993..

[22] Ruppen, H.-J. : Multiple TE-modes for cylindrical, self-focusing waveguides, pre-print.

[23] Ruppen, H.-J. : Multiple TE-modes for planar, self-focusing waveguides, pre-print. 
[24] Chen, Y. and Snyder, A. W. : TM-type self-guided beams with circular cross-section, Electr. Lett., 27 (1991), 565-566.

[25] Stuart, C .A. : Guidance properties of nonlinear planar waveguides, Arch. Rational Mech. Anal., 125 (1993), 145-200.

[26] Chen, Q. and Wang, Z. H. : Exact dispersion relations for TM waves guided by thin dielectric films bounded by nonlinear media, Opt. Letters, 18(1993), 260-262

[27] Kang, S.-W. : TM modes guided by nonlinear dielectric slabs, J. Lightwave Tech., 13(1995), 391-395

[28] Kushwaha, M. S. : Exact theory of nonlinear surface polaritons : TM case, Japanese J. Appl. Phys., 29(1995), 1826-1828

[29] Hiriart-Urruty, J. B. and Lemaréchal, C. : Convex Analysis and Minimisation Algorithms II, Springer-Verlag, Berlin, 1993

[30] Rockafellar, R. T. : Convex Analysis, Princeton Univ. Press, Princeton, 1970

[31] Landau, L. D., Lifshitz, E. M. and Pitaevskii, L.P. : Electrodynamics of Continuous Media, 2nd edition, Landau and Lifshitz Course of Theoretical Physics, Vol. 8, Pergamon Press, Oxford, 1984.

[32] Marburger, J. M. : Self-focusing, Theory, Prog. Quant. Electr., 4 (1975), $35-110$.

[33] Hayata, K., Koshiba, M. and Suzuki, M. : Finite-element solution of arbitrary nonlinear graded-index slab waveguides, Electr. Lttr., 23 (1987), 429-431.

[34] Chen, Y. : Effects of nonlinear saturation on vector spatial solitons, J. Opt. Soc. Am. B, 8 (1991), 2466-2469.

[35] Snyder, A. W. and Mitchell, D. J. : Spatial solitons of the power-law nonlinearity, Optics Letters, 18 (1993), 101-103.

[36] Wright, E. M., Heatley, D. R. and Stegeman, G. I. : Emission of spatial solitons from nonlinear waveguides, Physics Reports,194 (1990), 309323.

[37] Boardman, A. D. and Twardowski, T. : Theory of nonlinear interaction between TE and TM waves, J. Opt. Soc. Am. B, 5 (1988), 523-528. 
[38] Cuykendall, R. and Strobl, K. H. : Effect of soft saturation on nonlinear interface switching, Phy. Rev. A, 41 (1990), 352-358.

[39] Coutaz, J.-L. and Kull, M. : Saturation of the nonlinear index of refraction in semiconductor-doped glass, J. Opt. Soc. Am. B, 8 (1991), 95-98.

[40] Snyder, A. W. and Love, J. D. : Optical Wave Guide Theory, Chapman and Hall, London, 1983.

[41] Ruppen, H.-J. : Multiple cylindrical TM-modes for a homogeneous selffocusing dielectric, to appear in J. Diff. Equat..

ADDREss : Département de Mathématiques, EPFL, CH-1015 Lausanne, Switzerland 